\title{
Performance of the current and extended global NM network for solar particle registration and analysis
}

\author{
Alexander Mishev ${ }^{a, b, *}$ and Ilya Usoskin ${ }^{a, b}$ \\ ${ }^{a}$ Sodankylä Geophysical Observatory, \\ University of Oulu, Finland \\ ${ }^{b}$ Space Physics and Astronomy Research Unit, \\ University of Oulu, Finland \\ E-mail: alexander.mishev@oulu.fi, ilya.usoskin@oulu.fi
}

Over several decades, the global neutron monitor network was extensively and with undoubtful success used to study cosmic ray variations and fluxes of accelerated solar ions, the latter known as solar energetic particles. Recently, it has been used also for space weather purposes, specifically for alerts, and to provide crucial information necessary for assessment of different space weather effects, specifically the assessment of the exposure to radiation at flight altitudes. Here, we discuss the current status and applications of the global neutron monitor network, precisely its capability to study solar energetic particles, namely assessment of their spectral and angular distribution, during strong solar proton events e.g. those leading to ground level enhancements. Several examples are presented, accordingly. We also discuss the existing gaps in the network and propose an improvement of the network, namely a plan for an extension of the existing network with several new stations, in order to provide a more accurate analysis of strong solar proton events and to respond to the current space weather demands and services. We discuss the ability of the optimized global neutron monitor network to study various populations of solar energetic particles and to provide reliable space weather services.

$37^{\text {th }}$ International Cosmic Ray Conference (ICRC 2021)

July 12 th - 23rd, 2021

Online - Berlin, Germany

\footnotetext{
${ }^{*}$ Presenter
} 


\section{Introduction}

Cosmic rays (CRs) are flux of high-energy subatomic particles, including protons, $\alpha$-particles and heavier nuclei. Their energy ranges from about $10^{6}$ to $10^{21} \mathrm{eV} /$ nucleon, following roughly a power-law spectrum. The omnipresent part originate from the Galaxy, called galactic cosmic rays (GCRs), which is produced during and/or following supernova explosions. While the lowenergy $\mathrm{CR}$ particles are absorbed in the upper atmosphere, those with energies about $\mathrm{GeV} /$ nucleon produce secondary particles via interactions with the atmospheric constituents. Those secondaries also collide with atmospheric constituents, in turn producing other particles, if their energy is sufficiently high. Each collision adds a certain amount of particles, leading to the development of a complicated nuclear-electromagnetic-meson cascade known as an extensive air shower.

A sporadic source of high-energy particles, occasionally penetrating the Earth's atmosphere follows solar eruptions, viz. solar flares, and coronal mass ejection (CMEs), where solar ions can be accelerated to high energies, that is solar energetic particles (SEPs) [1]. When the energy of SEPs is about GeV/nucleon or greater, similarly to the GCRs, they produce a cascade of secondary particles in the Earth's atmosphere, that reaches the ground and increases the count rates of groundbased detectors, such as neutron monitors (NMs) [2]. This special class of SEP events is called ground-level enhancements (GLEs) [3, 4].

Accelerated to high energy solar ions lead to various space weather effects such as solar array performance degradation, glitches due to harm on electronic components in space missions or single event effects, threat to astronauts and/or aircrews over transpolar flights [5]. Therefore, SEPs, including GLE particles represent a specific and important space weather issue [6].

SEPs can be conveniently measured by space-borne instruments. Yet, the majority of the spaceborne instruments are constrained in the weight and size of the detector(s) and orbit in regions with a high rigidity cut-off. Therefore, space probes are not very suitable for the study of SEPs. On the other hand, GLEs can be studied using the worldwide NM network [7].

Here, we propose an extension of the global NM network with several new detectors. The aim is to optimize its performance, specifically for alerts connected with space weather purposes as well as to fill the existing gaps and improve the ability to register and analyze various populations of SEPs.

\section{Registration and analysis of GLEs using NMs}

Registration of a GLE can provide an early alert for the onset of SEP event [8,9]. Naturally, the alert systems require good coverage of the arrival direction of GLE particles by the global NM network since a selected NM stations shall exhibit a count rate increase. Besides, the spectral and angular characteristics of GLEs in the energy range $\sim 0.3-20 \mathrm{GeV} /$ nucleon, can be derived by modeling of the global NM network response and corresponding unfolding procedure using records form certain number of NM stations [3].

Methods for an analysis of GLEs using NM data have been developed over the years. They are based on modeling of the global NM network response and unfolding $n$ model parameters over the experimental records of $m$ NMs [3, 10, 11].

The relative count rate increase of a given NM during GLE can be modelled using: 


$$
\frac{\Delta N\left(P_{\mathrm{cut}}\right)}{N(t)}=\frac{\sum_{i} \sum_{k} \int_{P_{\mathrm{cut}}}^{P_{\max }} J_{\mathrm{sep}_{\mathrm{i}}}(P, t) S_{i, k}(P) G_{i}(\alpha(P, t)) A_{i}(P) d P}{\sum_{i} \int_{P_{\mathrm{cut}}}^{\infty} J_{\mathrm{GCR}_{\mathrm{i}}}(P, t) S_{i}(P) d P}
$$

where $N$ is the count rate due to GCR, $\Delta N\left(P_{\text {cut }}\right)$ is the count rate increase due to solar particles. $J_{\text {sep }}$ is the rigidity spectrum of SEPs $i$ (proton or $\alpha$-particle), $J_{\mathrm{GCR}_{\mathrm{i}}}(P, t)$ is the rigidity spectrum of the $i$ component (proton or $\alpha$-particle, etc...) of GCR at given time $t, G(\alpha(P, t))$ is the pitch angle distribution, note for GCRs the angular distribution is assumed to be isotropic, $\mathrm{A}(\mathrm{P})$ is a discrete function with $A(P)=1$ for allowed trajectories and $A(P)=0$ for forbidden trajectories. Function $A$ is derived during the asymptotic cone computations. $P_{\text {cut }}$ is the minimum rigidity cut-off of the station, accordingly, $P_{\text {cut }}$ is the maximum rigidity of SEPs considered in the model, whilst for GCR $P_{\max }=$ $\infty$. $S_{k}$ is the NM yield function for vertical and for oblique incidence SEPs. The contribution of oblique SEPs to NM response is particularly important for modeling strong and/or very anisotropic events, while for weak and/or moderately strong events it is possible to consider only vertical ones and using $S_{k}$ for an isotropic case, which considerably simplifies the computations [12].

The modeling of the global network NM response is carried out employing recently computed and validated yield function, [13-15]. The optimization is performed over the set of $n$ model parameters by minimizing the difference between the modeled and $m$ measured NM responses, that is by an inverse problem solution [16-20]. The main criterion for the optimization, that is the merit function is defined as:

$$
\mathcal{D}=\frac{\sqrt{\sum_{i=1}^{m}\left[\left(\frac{\Delta N_{i}}{N_{i}}\right)_{\text {mod. }}-\left(\frac{\Delta N_{i}}{N_{i}}\right)_{\text {meas. }}\right]^{2}}}{\sum_{i=1}^{m}\left(\frac{\Delta N_{i}}{N_{i}}\right)_{\text {meas }}}
$$

where $m$ is the number of NM stations, $\frac{\Delta N_{i}}{N_{i}}$ is the relative NM count rate increase for the $i$ NM station.

A robust and steady optimization and reliable solution are achieved when $\mathcal{D} \leq 5 \%$, a criterion usually fulfilled for strong events, whilst for moderately strong and weak events $\mathcal{D}$ can be about 10 or even up to $15 \%$. We emphasize that a solution can be obtained even in the case of $\mathcal{D} \sim 20-30$ $\%$, though resulting on greater uncertainties. Hence, it is necessary to possess about 2(n-1) data (NM stations), $n$ is the number of unknowns in the model, in order to be able to unfold the model parameters, therefore it is sufficient to retrieve information from 15-20 NMs, specifically those in a polar region, whilst the mid-latitude stations provide the necessary boundary conditions of the inverse problem.

The existing global NM network give a reliable basis to study GLEs, though a gap in the asymptotic directions of Arctic NMs is revealed Fig.1. One can see that South polar NMs provide relatively good coverage of the sky, whilst those at North exhibit gaps. Thus, if a GLE with narrow PAD occurs with anisotropy axis located in the polar region of the northern hemisphere, it would not be registered by the existing NMs (the black solid lines in Fgi.1). 


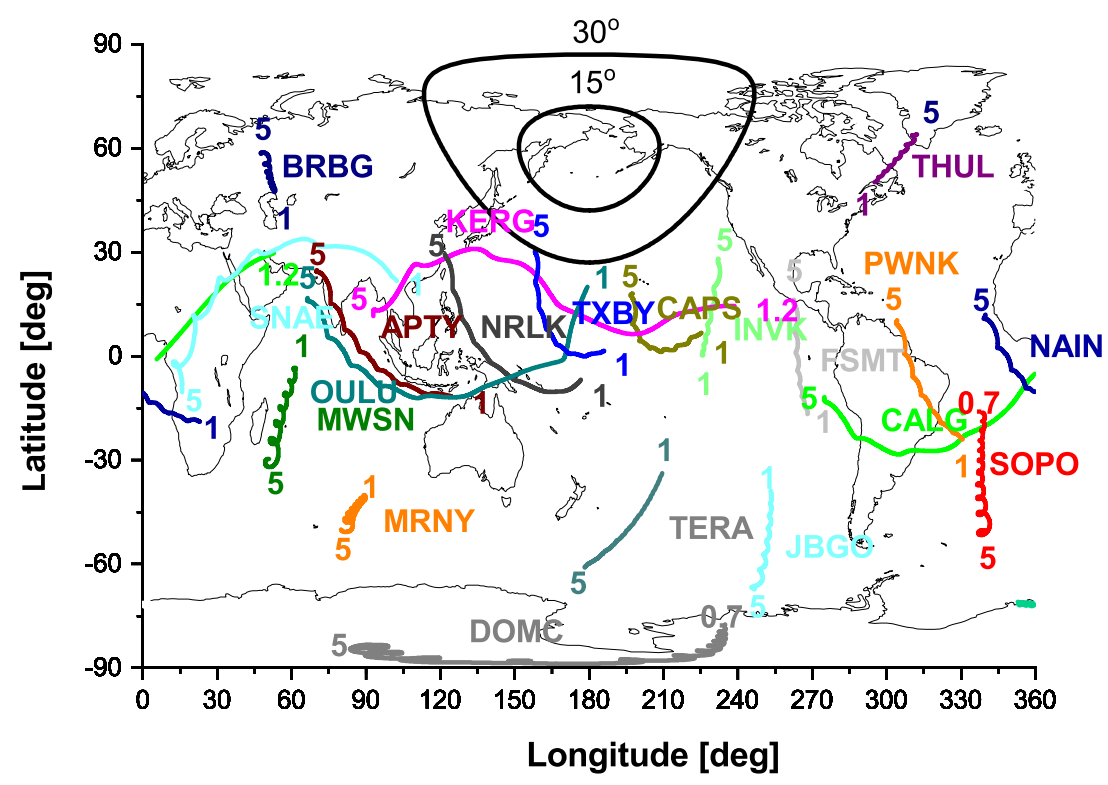

Figure 1: Asymptotic directions of polar NMs with the standard acronyms. The color lines depict asymptotic directions plotted in the rigidity range $1-5 \mathrm{GV}$, for DOMC, SOPO from 0.7 to $5 \mathrm{GV}$ respectively. The contour plot (black solid lines) for for $15^{\circ}$ and $30^{\circ}$, reveal the gap in the coverage.

\section{Extension of the global NM network}

The gap can be filled, by an extension of the NM network with several new stations: Severnaya Zemlya (SEVZ), Summit station in Greenland (SUMT) (for details see Fig. 2 and Table 1) and by reopening of the presently non-operational, but previously existed NMs: Alert (ALRT), Heis Island (HEIS) and Vostok (VSTK), the latter for optimization the performance of the unfolding procedure. One can see that an extended network of polar stations would provide almost global coverage in the maximal NM response rigidity range of 1-5 GV. Besides, in order to improve the sensitivity of the global NM network for registration of solar neutrons [21], we propose to several new low-latitude stations (Table 1). Summary of the extension of the global network is given in Table 1 and Fig.3.

Table 1: Extension of the NM network. Columns represent station name, location, geomagnetic cut-off rigidity and altitude above sea level. The part above the double line corresponds to the closed but previously existing stations to be reopened, the bottom part corresponds to the new stations.

\begin{tabular}{ccccc} 
Station & latitude $[\mathrm{deg}]$ & Longitude [deg] & $P_{c}[\mathrm{GV}]$ & Altitude [m] \\
\hline Alert (ALRT) & 82.5 & 297.67 & 0.0 & 57 \\
Heiss island (HEIS) & 80.62 & 58.05 & 0.1 & 20 \\
Haleakala (HLEA) & 20.71 & 203.74 & 12.91 & 3052 \\
Vostok (VSTK) & -78.47 & 106.87 & 0.0 & 3488 \\
\hline \hline Canary Islands (CANI) & 28.45 & 342.47 & 11.76 & 2376 \\
New Zealand (NZLD) & -43.59 & 170.27 & 3.28 & 1029 \\
Severnaya Zemlya (SEVZ) & 79.29 & 96.5 & 0.11 & 10 \\
Summit (SUMT) & 72.34 & 321.73 & 0.01 & 3126 \\
\hline
\end{tabular}




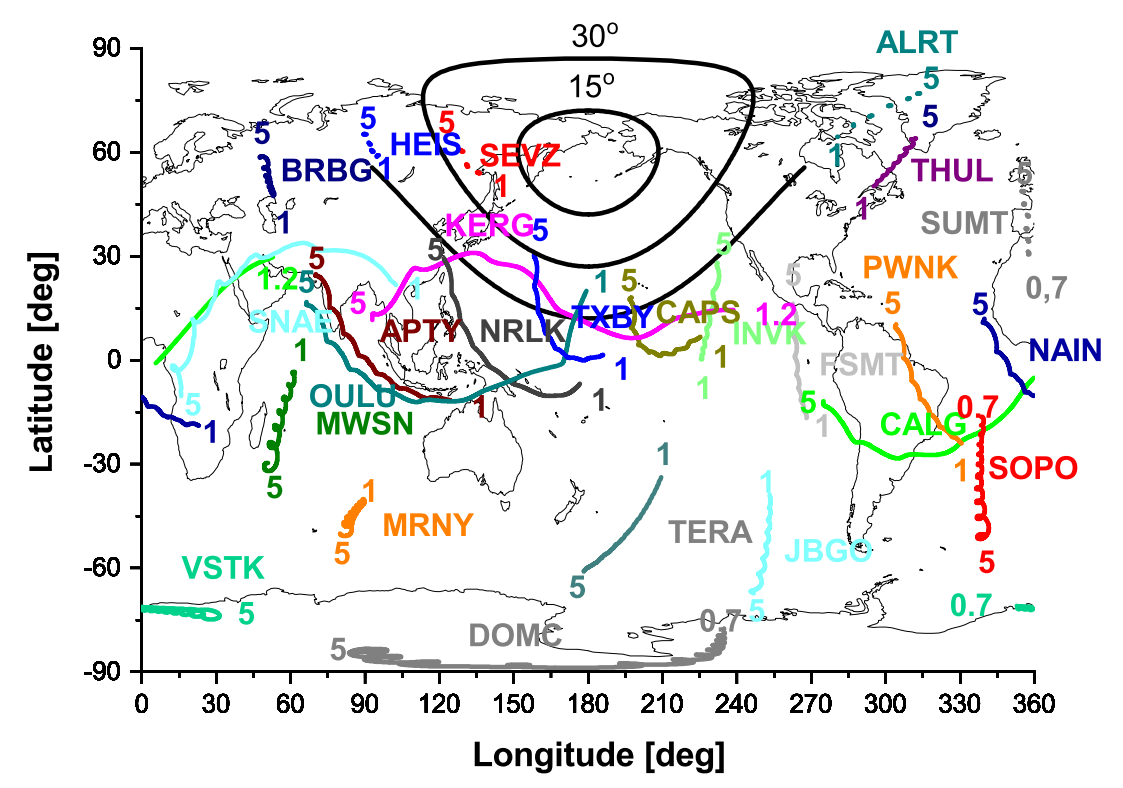

Figure 2: The same as Fig.1. The dashed lines correspond to new NMs proposed for extension of the network or to be reopened.

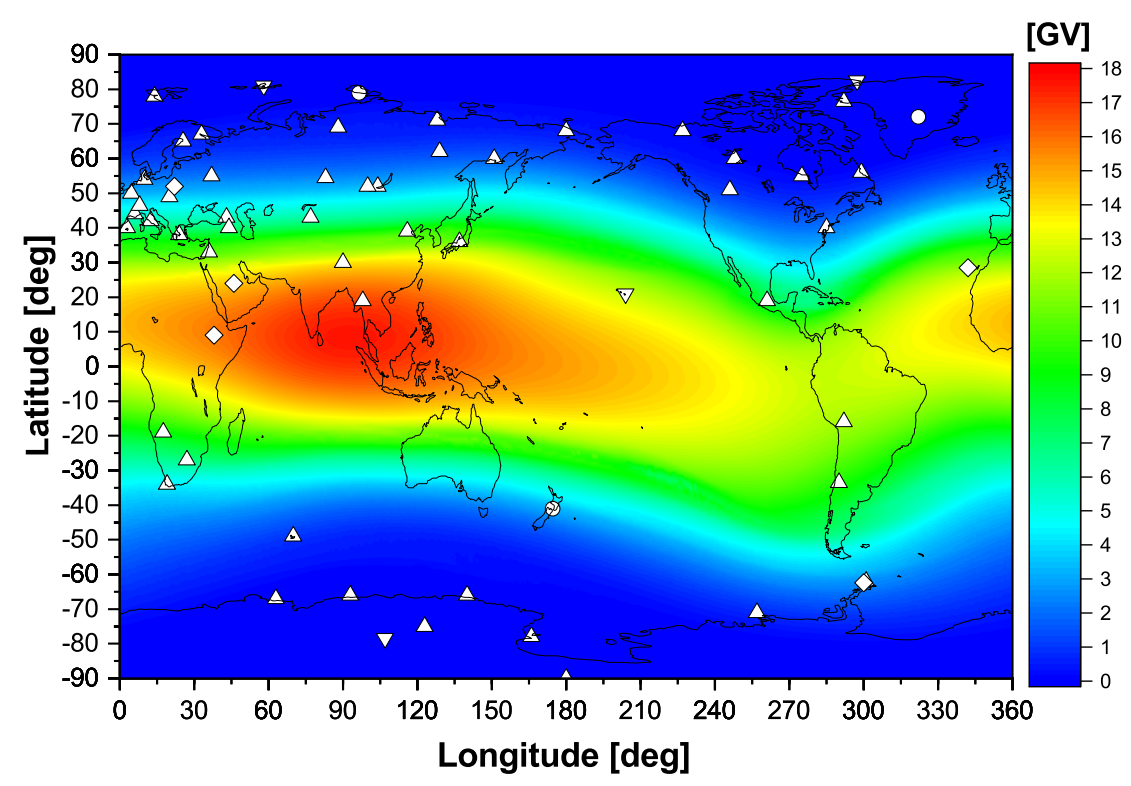

Figure 3: Global NM network. The upper triangles depict the existing stations, lower triangles correspond the previously existing stations to be reopened, circles to the new stations, rhombus to planned or under construction stations. 


\section{Performance of extended NM network for analysis of GLEs}

We studied the performance for GLE analysis employing the procedure described in Section 2, considering different number of NM records. Thus, we studied the performance of the extended, actual and reduced (in case of closure of stations) NM network, the details are given in Table 2. One can see that the extended NM network would result in considerably smaller $\mathcal{D}$ compared to the actual number of NMs used for the analysis, whilst a reduction of the number of NMs leads to jeopardizing the ability of the global NM network to provide a reliable GLE analysis. Here, the data from additional NMs are obtained using forward modelling employing the actual derived SEP spectra and PADs [12, 18, 20, 22, 23].

Table 2: The value of $\mathcal{D}$ during the main phase of selected GLEs as a function of the number of the used NMs. Columns 1-2 correspond to the number and date of the GLE, while columns 3-5 correspond to $\mathcal{D}$ and number of the used stations (in the brackets) for extended NM network, actual NM network used for the analysis and the reduced NM network, respectively. N.A. corresponds to the case when the SEP spectra cannot be unfolded.

\begin{tabular}{cc|ccc} 
GLE \# & Date & Extended NM network & Actual NM network & Reduced NM network \\
\hline GLE \# 59 & 14.07 .2000 & $4.1(39)$ & $4.8(30)$ & $19(20)$ \\
GLE \# 67 & 02.11 .2003 & $4.5(39)$ & $7.1(34)$ & $38(21)$ \\
GLE \# 69 & 20.01 .2005 & $3.0(38)$ & $3.5(33)$ & $35(25)$ \\
GLE \# 70 & 13.12 .2006 & $3.2(38)$ & $4.2(32)$ & $43(22)$ \\
GLE \# 71 & 17.05 .2012 & $5.0(34)$ & $7.1(24)$ & N.A.(19) \\
GLE \# 72 & 10.09 .2017 & $5.2(31)$ & $6.1(23)$ & $33(18)$
\end{tabular}

\section{Conclusions}

Here we discussed the ability of the global NM network to register and provide reliable information for GLE analysis. A gap of the current network is clearly seen, therefore we proposed an extension of the network with several new stations, that is, we propose to reopen four previously operational NMs: ALRT, HEIS, HLEA and VSTK and to build four new stations: CANI, NZLD, SEVZ, SUMT. Note, that CANI NM is under construction. Besides, we demonstrated the performance of the extended NM network for GLE analysis. It was shown that data from the extended network result on significant improvement of GLE analysis, whilst the reduction would lead to deterioration of the derived information. This study is a continuation of the previous proposition for optimization of the global NM network [24]. Here, we would like to stress that even a partial reduction of the number of existing NMs would considerably influence the capability of the global NM network GLEs registration and the corresponding space weather services [25]. Nowadays, the existence and continuous functioning of several NM stations is questioned, therefore, the support of the network from governments, foundations(s) and space flight operators is crucially needed.

\section{Acknowledgements}

This work was supported by the Academy of Finland (project 330064 QUASARE and 321882 ESPERA). We acknowledge the PIs and colleagues from NM stations used in this study. 


\section{References}

[1] M. Desai and J. Giacalone, Large gradual solar energetic particle events, Living Reviews in Solar Physics 13 (2016), no. 13.

[2] M. Aschwanden, GeV particle acceleration in solar flares and ground level enhancement (GLE) events, Space Science Reviews 171 (2012), no. 1-4 3-21.

[3] M. Shea and D. Smart, Possible evidence for a rigidity-dependent release of relativistic protons from the solar corona, Space Science Reviews 32 (1982), 251-271.

[4] S. Poluianov, I. Usoskin, A. Mishev, A. Shea, and D. Smart, GLE and sub-GLE redefinition in the light of high-altitude polar neutron monitors, Solar Physics 292 (2017), no. 11176.

[5] R. Vainio, L. Desorgher, D. Heynderickx, M. Storini, E. Flückiger, R. Horne, G. Kovaltsov, K. Kudela, M. Laurenza, S. McKenna-Lawlor, H. Rothkaehl, and I. Usoskin, Dynamics of the Earth's particle radiation environment, Space Science Reviews 147 (2009), no. 3-4 187-231.

[6] A. Mishev, and P. Jiggens, Preface to measurement, specification and forecasting of the Solar Energetic Particle (SEP) environment and Ground Level Enhancements (GLEs), Journal of Space Weather and Space Climate 9 (2019), E1.

[7] J. Simpson. The Cosmic Ray Nucleonic Component: The Invention and Scientific Uses of the Neutron Monitor, Space Science Reviews 93 (2000), 11-32.

[8] T. Kuwabara, J. Bieber, J. Clem, P. Evenson, and R. Pyle. Development of a ground level enhancement alarm system based upon neutron monitors. Space Weather 4 (2006), no. 10, S10 001 .

[9] H. Mavromichalaki, H., M. Gerontidou, P. Paschalis, E. Paouris, A. Tezari, C. Sgouropoulos, N. Crosby, and M. Dierckxsens, Real-Time Detection of the Ground Level Enhancement on 10 September 2017 by A.Ne.Mo.S.: System Report Space Weather, 16 (2018), 1797-1805.

[10] J. Cramp, M. Duldig, E. Flückiger, J. Humble, M. Shea and D. Smart, The October 22, 1989,solar cosmic enhancement: ray an analysis the anisotropy spectral characteristics, Journal of Geophysical Research 102 (1997), no. A11 24 237-24 248.

[11] E. Vashenyuk, Y. Balabin, J. Perez-Peraza, A. Gallegos-Cruz and L. Miroshnichenko, Some features of the sources of relativistic particles at the sun in the solar cycles 21-23, Advances Space Research 38 (2006), no. 3 411-417.

[12] A. Mishev and I. Usoskin, Analysis of the ground level enhancements on 14 July 2000 and on 13 December 2006 using neutron monitor data, Solar Physics 291 (2016), no. 4 1225-1239.

[13] A. Mishev , I. Usoskin, and G. Kovaltsov. Neutron Monitor Yield Function: New Improved computations. J. Geophys. Res. (Space Phys.), 118, (2013), 2783-2788. 
[14] S.A. Koldobskiy, , V. Bindi, C. Corti, G. A. Kovaltsov, and I. G. Usoskin. Validation of the Neutron Monitor Yield Function Using Data from AMS-02 Experiment 2011-2017. J. Geophys. Res. (Space Phys.), 124, (2019) 2367-2379

[15] A.L. Mishev, S.A. Koldobskiy, G.A. Kovaltsov, A. Gil, and I.G. Usoskin. Updated NeutronMonitor Yield Function: Bridging Between In Situ and Ground-Based Cosmic Ray Measurements. J. Geophys. Res. (Space Phys.), 125 (2020), e2019JA027,433.

[16] A. Tikhonov, A. Goncharsky, V. Stepanov and A. Yagola, Numerical Methods for Solving ill-Posed Problems. Kluwer Academic Publishers, Dordrecht, 1995.

[17] S. Mavrodiev, A. Mishev and J. Stamenov, A method for energy estimation and mass composition determination of primary cosmic rays at the Chacaltaya observation level based on the atmospheric Cherenkov light technique, Nucl. Instr. and Methods in Phys. Res. A 530 (2004), no. 3 359-366.

[18] A. Mishev, L. Kocharov, and I. Usoskin, Analysis of the ground level enhancement on 17 May 2012 using data from the global neutron monitor network, Journal of Geophysical Research 119 (2014) 670-679.

[19] A. Mishev, S. Poluianov and I. Usoskin, Assessment of spectral and angular characteristics of sub-GLE events using the global neutron monitor network, Journal of Space Weather and Space Climate 7 (2017) A28.

[20] A. Mishev, I. Usoskin, O. Raukunen, M. Paassilta, E. Valtonen, L. Kocharov and R. Vainio, First analysis of GLE 72 event on 10 September 2017: Spectral and anisotropy characteristics, Solar Physics 293 (2018) 136.

[21] A. Artamonov, G. Kovaltsov, A. Mishev, and I. Usoskin. Neutron monitor yield function for solar neutrons: A new computation., Journal of Geophysical Research A: Space Physics 121 (2016), 117-128.

[22] L. Kocharov, S. Pohjolainen, A. Mishev et al., Investigating the origins of two extreme solar particle events: Proton source profile and associated electromagnetic emissions, The Astrophysical Journal 839 (2017), no. 279.

[23] A. Mishev, S. Koldobskiy, L. Kocharov and I. Usoskin, GLE \# 67 Event on 2 November 2003: An Analysis of the Spectral and Anisotropy Characteristics Using Verified Yield Function and Detrended Neutron Monitor Data, Solar Physics in press (2021).

[24] J. Bieber and P. Evenson, Spaceship earth - an optimized network of neutron monitors, in Proc. of 24th ICRC Rome, Italy, 28 August - 8 September 1995, vol. 4, pp. 1316-1319, 1995.

[25] A. Mishev and I. Usoskin, Current status and possible extension of the global neutron monitor network, J. Space Weather Space Clim. 10 (2020), 17. 